\title{
Influence of soil properties on plant density and species richness of saline desert
}

Pradeep Kumar Pilania \& Nilesh Sundarjibhai Panchal

Department of Biosciences, Saurashtra University, Rajkot-360005,Gujarat, India.

\section{Resumen}

Correspondence

N.S. Panchal

E-mail: nspanchal@live.com

Received: 8 January 2016

Accepted: 29 April 2016

Published on-line: 7 June 2016
Influencia de las propiedades del suelo en la densidad de plantas y la riqueza de especies de desierto salino

El ambiente edáfico tiene una significativa influencia en la productividad. La salinidad es uno de los principales factores que afectan negativamente a la vegetación. Se estudió un ecosistema desértico de la India (Pequeño Rann de Kutch; 7020 ha) para medir la influencia de las propiedades del suelo en la vegetación. Tanto la riqueza de especies (SR) como la densidad de hierbas y arbustos/árboles (17.018 plantas $\mathrm{m}^{-2}$ y 8.617 plantas $10 \mathrm{~m}^{-2}$ ) fueron mayores en el punto 4, con valores altos de OC, OM, N, P, Ca y Fe $\left(0.684,1.179,0.059 \%, 42.338 \mathrm{~kg} \mathrm{ha}^{-1}, 170.732,32.016 \mathrm{mg} \mathrm{kg}^{-1}\right)$ y bajos niveles de arcilla, EC y Na $\left(33.654 \%, 9.441 \mathrm{dSm}^{-1}\right.$ y 68.699 $\mathrm{mg} \mathrm{kg}^{-1}$ ). Valores altos de arcilla, $\mathrm{Na}$ y EC con bajo $\mathrm{Ca}$ y Fe resultaron en bajas densidades de SR (lugares 2 y 5). Las bajas SR y densidad se deben a bajas concentraciones de OC, OM, N, P, Fe, Ca y alta concentración de arcilla, Na y EC.

Palabras clave: Calcio, Desierto, Salinidad, Suelo, Sodio, Vegetación.

\begin{abstract}
Soil environment have significant influence on the productivity of land. Salinity is one of the major factors which negatively affect the vegetation. To measure the influence of soil properties on vegetation, desert ecosystem in India (Little Rann of Kutch of 7020 ha) was studied. Species richness (SR) as well as density for herbs and shrubs/tree (17.018 plants $\mathrm{m}^{-2}$ and 8.617 plants $10 \mathrm{~m}^{-2}$ ) was highest in the site 4, with high $\mathrm{OC}, \mathrm{OM}, \mathrm{N}, \mathrm{P}, \mathrm{Ca}$ and $\mathrm{Fe}(0.684$, $1.179,0.059 \%, 42.338 \mathrm{~kg} \mathrm{ha}^{-1}, 170.732$ and $32.016 \mathrm{mg} \mathrm{kg}^{-1}$ ) and low clay, EC and $\mathrm{Na}\left(33.654 \%, 9.441 \mathrm{dSm}^{-1}\right.$ and $\left.68.699 \mathrm{mg} \mathrm{kg}^{-1}\right)$. High amount of clay, $\mathrm{Na}$ and $\mathrm{EC}$ with low $\mathrm{Ca}$ and $\mathrm{Fe}$ results into low density with low SR (site 2 and 5). Low SR and density are due to low concentration of $\mathrm{OC}, \mathrm{OM}, \mathrm{N}, \mathrm{P}, \mathrm{Fe}, \mathrm{Ca}$ and high concentration of clay, $\mathrm{Na}$ and $\mathrm{EC}$.
\end{abstract}

Key words: Calcium, Desert, Salinity, Soil, Sodium, Vegetation. 


\section{Introduction}

Soil salinization and sodification had been identified as a major cause of land degradation. Salt-affected areas increase at a high rate, by about two million hectares per year (Postel 1996). Soil and vegetation together are vital factors of any ecosystem. The risk of soil degradation depends on the total salt content and on the salt composition; especially in relation to sodium concentration.

Salinization is the augment of the soluble salt in the root zone of the soil while sodification is the increase of exchangeable sodium in the root zone of the soil. The two processes may operate concurrently and form saline-sodic soils. The distribution is relatively more extensive in the arid and semi-arid regions. Soil is a natural resource that is not renewable within a point of petite time scale. Responsiveness of erosion extent and intensity for determining principal strategies and most encouraging soil conservation, as well as control of erosion and sediment yield are matters of concern for researchers, so that they can envisage the spatial pattern and erosion hazards rates (Morgan 1996). The division of plant species in saline soil is closely related with soil water potentials and factors controlling the level of salinity stress, including precipitation, depth of the water table (Ungar et al. 1979). Soils on landscape surfaces and good plant cover conditions may recover with time by accruing organic material, increasing floral and faunal activity, enhancing soil aggregate stability, increasing infiltration capacity, and decreasing erosion potential (Trimble 1990).

Vegetation cover is the chief factor to control soil degradation by water and wind erosion, the efficiency varies greatly with vegetation type and land cover. Soil erosion is expected to be more affected by changes vegetation cover than by runoff (Nearing et al. 2005).

The effect of vegetation on soil parameters have been known since the development of the concept of the factors of soil formation (Jenny 1941). Vegetation influences the soil by recycling different nutrients, which suggests that to increase the productivity of the land both soil and vegetation should be studied simultaneously.

The major goal of this study was to understand the inter-relation of soil (physical and chemical properties) and vegetation of the saline desert. Little Rann of Kutch is highly saline and salinity has negative effects on the vegetation except some salt tolerant species for example Prosopis juliflora (Sw.) DC., Acacia nilotica (Linn.), Salvadora oleoides Decne., Aeluropus lagopoides (Linn.), Cressa cretica Linn. etc. Salt stress is a worldwide problem, but is of special concern in arid and semi-arid regions. High concentrations of salts have harmful effects on plant growth (Mer et al. 2000; Vaghasiya et al. 2015) and excessive concentrations kill growing plants (Donahue et al. 1983). Many investigators have reported retardation of germination and growth of seedlings at high salinity (Garg and Gupta 1997).

Soil and vegetation degradation both are influenced by each other reduction in the perennial cover or vegetation cover is regarded as an indicator of the onset of desertification (Thornes 1996). All kinds of cover that secure against the erosive elements such as runoff, raindrop impacts and wind referred to as land cover. Type of land cover includes vegetation, stone, litter and gravel covers. Generally unnatural land use that diminishes the amount of land cover on an incline may cause severe erosion and sediment construction (Refahi 2006). With this alarm our aim was to study inter-relation of soil and vegetation and to identify the effect of different soil properties on plant density and species richness at the saline desert of western India soil.

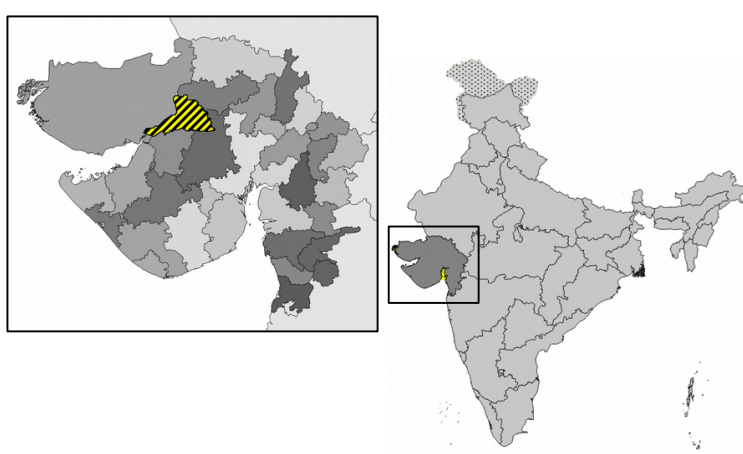

Figura 1. Zona de estudio en el Pequeño Rann de Kutch. Figure 1. Study Area in Little Rann of Kutch.

\section{Material and methods}

\section{Study Area}

The study was conducted in India at Little Rann of Kutch (22 $55^{\prime \prime}$ to $24^{\circ} 35^{\prime \prime}$ North latitudes and 
$70^{\circ} 30^{\prime \prime}$ to $71^{\circ} 45^{\prime \prime}$ East longitudes) known as "The Wild Ass Sanctuary", named after endangered ghudkhur (Equs hemionus khur Lesson, 1827). The Little Rann of Kutch (Fig. 1) occupies an area of $6979 \mathrm{sq} \mathrm{km}$ of which the Wild Ass Sanctuary encompassing $4953 \mathrm{sq} \mathrm{km}$

\section{Soil Analysis}

\section{Collection of soil samples}

Collections of samples were done in the months of mid June to October (2014). To represent the harsh condition of this area the ombro-thermic diagram is given (Fig. 2). Samples were randomly collected from five different sites $(20,16,20,64$ and 36 samples from site one to five respectively), for three depths i.e., $0-15,15-30$ and 30-45 cm respectively. Soil samples were thoroughly mixed depth wise, and from the composite soil, one sample was drawn for each depth and brought to the laboratory. All these soil samples were air dried and stored in polyethylene bags to determine their physical and chemical properties. For soil aggregate analysis, soil samples were collected separately from each site. Due care was taken, specially, in sampling and in transportation to the laboratory, so that the soil aggregate should not be disturbed.

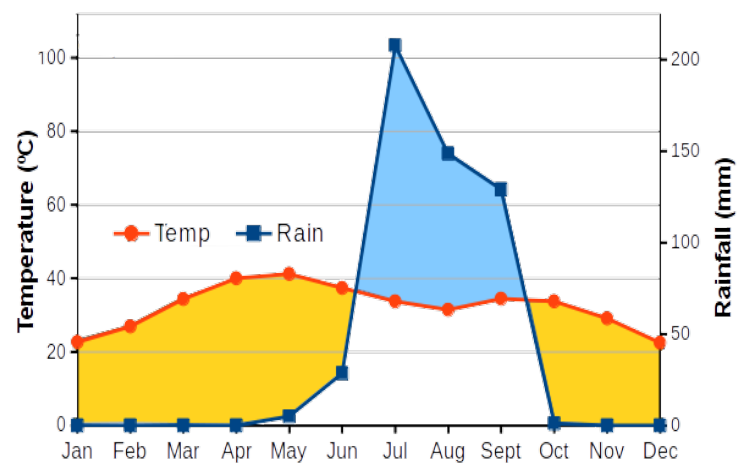

Figura 2. Diagrama ombrotémico del área de estudio, basado en los datos de temperatura y precipitaciones de diez años (2004 2014).

Figure 2. Ombro-thermic diagrams for study area based on temperature and rainfall data for ten years (2004 to 2014).

\section{Analysis of Physical Properties of Soil}

Soil Texture was determined by "Bouyoucos Hydrometer Method" (Bouyoucos 1951). Soil Aggregates was determined by "Wet sieving method" (Yoder 1936) with the help of a Yoder sieve shaker. Soil weight in unit volume was computed to determine bulk density (BD). Particle density (PD) was measured by method given by USDA (Richards 1968). Value of bulk density was used to determine porosity (PO) of soil (Misra 1968) and expressed in percentage.

\section{Soil Moisture Constants}

Field Capacity (FC) and water holding capacity (WHC) was determined following Misra (1968) and the results are expressed in percentage of oven-dry weight (Oven-drying was done at $105^{\circ} \mathrm{C}$ temperature).

\section{Analysis of Chemical Properties of Soil}

Soil $\mathrm{pH}$ was measured by $\mathrm{pH}$ meter preparing soil paste with distilled water (1:5 ratio). Electrical Conductivity (EC) was measured by an EC meter (1:2 ratio). As per Jackson (1973), Organic carbon $(\mathrm{OC})$, Organic matter $(\mathrm{OM})$ and Nitrogen $(\mathrm{N})$ were measured by using UV Visible spectrophotometer. Available Phosphorus (P) was measured by the method of Olsen et al. (1954). As per Lindsay and Norvell 1978, Potassium (K), Sodium (Na), Calcium $(\mathrm{Ca})$, Zinc $(\mathrm{Zn})$, Copper $(\mathrm{Cu})$, Iron $(\mathrm{Fe})$, Lead $(\mathrm{Pb})$ and Manganese $(\mathrm{Mn})$ were measured by Atomic Absorbance Spectrophotometer.

\section{Vegetation analysis}

Vegetation was quantitatively analyzed for density following Curtis and McIntosh (1950). Species richness (SR) was calculated as per Margalef 1958.

\section{Results}

\section{Characterization of physical properties of soil}

BD was maximum (Table 1) at site one $(2.080$ $\left.\mathrm{gcc}^{-1}\right)$ while minimum at site four $\left(1.765 \mathrm{gcc}^{-1}\right)$. PD was highest at site two $\left(3.313 \mathrm{gcc}^{-1}\right)$ while lowest at site five $\left(2.612 \mathrm{gcc}^{-1}\right)$. At each site BD and PD was maximum at lower depths except at site two. PO maximum values obtained at upper layer of soil at sites one, three and four. FC and WHC were determined to know the soil moisture content of the soil and expressed in percentage of oven-dry weight. FC was highest at site four $(22.935 \%)$ while minimum at site one $(20.114 \%)$ and $\mathrm{WHC}$ was highest (37.152\%) at site one and lowest $(24.254 \%)$ at site four. 


\begin{tabular}{|c|c|c|c|c|c|c|c|}
\hline & \multicolumn{2}{|c|}{ Soil Depth } & Site 1 & Site 2 & Site 3 & Site 4 & Site 5 \\
\hline \multirow{5}{*}{$\begin{array}{c}\text { BD } \\
\left(g^{\prime} c c^{-1}\right)\end{array}$} & \multirow{3}{*}{\multicolumn{2}{|c|}{$\begin{array}{c}0-15 \\
15-30 \\
30-45\end{array}$}} & $2.065 \pm 0.075$ & $146 \pm 0.0$ & $853 \pm 0$. & \pm 0 . & \pm 0. \\
\hline & & & \pm 0.085 & $2.011 \pm 0.043$ & $1.961 \pm 0.036$ & \pm 0.045 & \pm 0.068 \\
\hline & & & $2.127 \pm 0.058$ & $1.929 \pm 0.049$ & $1.931 \pm 0.029$ & \pm 0.047 & \pm 0.068 \\
\hline & \multirow{2}{*}{\begin{tabular}{|c|} 
Combined \\
$0-45$
\end{tabular}} & an & $2.080 \pm 0.041$ & $2.029 \pm 0.034$ & $1.915 \pm 0.022$ & \pm 0.028 & $1.818 \pm 0.040$ \\
\hline & & & 1.380 to 2.843 & 1.700 to 2.409 & 1.522 to 2.088 & 1.128 to 2.303 & 1.280 to 2.294 \\
\hline \multirow{5}{*}{$\begin{array}{c}\text { PD } \\
\left(\mathrm{gcc}^{-1}\right)\end{array}$} & & $3.008 \pm 0.188$ & $3.695 \pm 0.415$ & $3.091 \pm 0.240$ & \pm 0.106 & \pm 0.106 \\
\hline & & & $2.734 \pm 0.223$ & $2.940 \pm 0.081$ & $3.199 \pm 0.213$ & \pm 0.106 & \pm 0.051 \\
\hline & & & $3.081 \pm 0.204$ & $3.305 \pm 0.201$ & $2.978 \pm 0.253$ & $2.618 \pm 0.120$ & $\pm \quad 0.119$ \\
\hline & \multirow{2}{*}{$\begin{array}{c}\text { Combined } \\
0-45\end{array}$} & ean & $2.941 \pm 0.116$ & $3.313 \pm 0.156$ & $3.089 \pm 0.131$ & $2.679 \pm 0.063$ & \pm 0.055 \\
\hline & & $\mathrm{R}$ & 1.642 to 4.926 & 2.008 to 5.814 & 1.799 to 4.329 & 1.597 to 4.762 & 1.783 to 4.831 \\
\hline \multirow{5}{*}{$\begin{array}{l}\text { PO } \\
\text { (\%) }\end{array}$} & \multicolumn{2}{|c|}{$0-15$} & $568 \pm 3.478$ & $35.864 \pm 5.265$ & $35.974 \pm 3.965$ & $33.936 \pm 2.213$ & $29.689 \pm$ \\
\hline & \multicolumn{2}{|c|}{$15-30$} & $24.453 \pm 2.790$ & $31.328 \pm 1.337$ & $35.230 \pm 3.895$ & $32.888 \pm 2.007$ & $32.083 \pm 2.011$ \\
\hline & \multicolumn{2}{|c|}{$30-45$} & $28.139 \pm 2.927$ & $41.090 \pm 4.290$ & $30.870 \pm 4.340$ & $28.628 \pm 2.138$ & $28.613 \pm 1.791$ \\
\hline & \multirow{2}{*}{$\begin{array}{c}\text { Combined } \\
0-45\end{array}$} & & $28.053 \pm 1.753$ & $36.094 \pm 2.277$ & $34.024 \pm 2.271$ & $31.817 \pm 1.223$ & $30.128 \pm 1$ \\
\hline & & ge & 10.190 to 58.625 & 17.846 to 66.364 & 13.012 to 55.194 & 10.005 to 62.460 & 15.435 to 67.369 \\
\hline & & & $18.644 \pm 1.033$ & $26.459 \pm 1.861$ & $22.115 \pm 0.481$ & $22.491 \pm 0.450$ & $20.259 \pm$ \\
\hline & & & $18.852 \pm 0.828$ & $22.862 \pm 1.548$ & $20.903 \pm 0.231$ & $21.692 \pm 0.575$ & $19.050 \pm 1$. \\
\hline FC & & & $22.846 \pm 1.561$ & $17.927 \pm 1.379$ & $20.797 \pm 1.405$ & $24.621 \pm 1.131$ & $21.595 \pm 1.241$ \\
\hline & & an & $20.114 \pm 0.712$ & $22.416 \pm 1.049$ & $21.272 \pm 0.487$ & $22.935 \pm 0.438$ & $20.301 \pm 0.643$ \\
\hline & & & 10.968 to 34.016 & 13.434 to 38.686 & 16.031 to 30.788 & 11.847 to 38.267 & 12.399 to 37.806 \\
\hline & & & $33.089 \pm 2.365$ & $26.467 \pm 0.464$ & $28.187 \pm 0.726$ & $24.622 \pm 0.549$ & $27.261 \pm 0.881$ \\
\hline & & & $40.578 \pm 2.552$ & $24.780 \pm 1.239$ & $28.125 \pm 0.553$ & $23.957 \pm 0.512$ & $27.443 \pm 1.521$ \\
\hline $\begin{array}{c}\text { WHC } \\
(\%)\end{array}$ & 30 & & $37.790 \pm 3.098$ & $25.103 \pm 0.844$ & $27.596 \pm 0.241$ & $24.183 \pm 0.463$ & $27.858 \pm 1.236$ \\
\hline & & & $37.152 \pm 1.553$ & $25.450 \pm 0.509$ & $27.969 \pm 0.303$ & $24.254 \pm 0.281$ & $27.520 \pm 0.699$ \\
\hline & & & 19.662 to 52.502 & 19.214 to 31.140 & 24.721 to 33.562 & 16.986 to 33.567 & 19.214 to 49.069 \\
\hline
\end{tabular}

Tabla 1. Descripción de propiedades físicas del suelo en diferentes lugares y profundidades. BD=Densidad aparente, $\mathrm{PD}=\mathrm{Den}$ sidad de partículas, $\mathrm{PO}=$ Porosidad, $\mathrm{FC}=\mathrm{Capacidad}$ de campo, WHC=Capacidad de carga hídrica; se proporcionan la media y rango, por separado, para profundidades de $0-45 \mathrm{~cm}$.

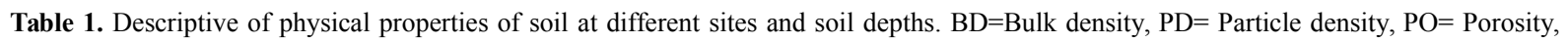
$\mathrm{FC}=$ Field capacity, $\mathrm{WHC}=$ Water holding capacity; separate mean and range is given for $0-45 \mathrm{~cm}$ depth .

\section{Characterization of soil texture and soil aggregate}

Site one, three and four contain clay loam type while site two and five contain clay type texture. Maximum values of sand (35.238\%), clay $(44.160 \%)$ and silt $(40.335 \%)$ was found at site four, two and one while minimum value for sand $(26.067 \%)$ and clay $(33.589 \%)$ at site one and for silt $(27.569 \%)$ at site two (Table 2). High amount of large macro-aggregate was at lower depths except at site three.

\section{Characterization of chemical properties of soil}

Correlation of $\mathrm{Na}$ and $\mathrm{Ca}$ was negative (-0.696) and positive correlation of $\mathrm{K}$ and $\mathrm{Zn}(0.737)$ (Fig. 3). $\mathrm{pH}$ (Table 3) was maximum at site one (9.036) while minimum at site five (7.796). EC was maximum at site two $\left(14.031 \mathrm{dSm}^{-1}\right)$ followed by site three $\left(13.171 \mathrm{dSm}_{-1}\right)$ while minimum at site four $\left(9.441 \mathrm{dSm}^{-1}\right)$. OC $(0.684 \%), \mathrm{OM}(1.179 \%), \mathrm{N}$ $(0.059 \%)$ and $\mathrm{P}\left(42.338 \mathrm{~kg} \mathrm{ha}^{-1}\right)$ were maximum at site four, while OC (0.605\%), OM (1.042\%) and $\mathrm{N}(0.052 \%)$ were minimum at site two and $\mathrm{P}$ $\left(36.031 \mathrm{~kg} \mathrm{ha}^{-1}\right)$ at site five. $\mathrm{K}$ was maximum at site one $\left(873.672 \mathrm{mg} \mathrm{kg}^{-1}\right)$ and minimum at site four $\left(53.118 \mathrm{mg} \mathrm{kg}^{-1}\right)$. Ca was maximum at site one and four (170.923 and $\left.170.732 \mathrm{mg} \mathrm{kg}^{-1}\right)$ while minimum at site two and five (51.989 and 91.120 $\left.\mathrm{mg} \mathrm{kg}^{-1}\right)$. Na was maximum at site two and five (166.149 and $141.128 \mathrm{mg} \mathrm{kg}^{-1}$ ) while minimum at site four and one (68.699 and $\left.72.380 \mathrm{mg} \mathrm{kg}^{-1}\right)$. $\mathrm{Zn}$ was maximum at site two and minimum at site one. $\mathrm{Cu}\left(22.460 \mathrm{mg} \mathrm{kg}^{-1}\right)$, Fe (48.053 $\left.\mathrm{mg} \mathrm{kg}^{-1}\right)$ and Mn (38.565 mg kg-1) were maximum at site three while $\mathrm{Cu}$ and $\mathrm{Fe}$ (18.061 and $19.577 \mathrm{mg} \mathrm{kg}^{-1}$ ) were minimum at site two and $\mathrm{Mn}(24.799 \mathrm{mg}$ $\mathrm{kg}^{-1}$ ) at site four. $\mathrm{Pb}$ was maximum at site five (48.843 $\mathrm{mg} \mathrm{kg}^{-1}$ ) and minimum at site three (42.932 $\left.\mathrm{mg} \mathrm{kg}^{-1}\right)$. 


\begin{tabular}{|c|c|c|c|c|c|c|c|}
\hline & \multicolumn{2}{|c|}{ Soil Depth } & Site 1 & Site 2 & Site 3 & Site 4 & Site 5 \\
\hline \multirow{5}{*}{$\begin{array}{c}\text { Sand } \\
(\%)\end{array}$} & \multirow{3}{*}{\multicolumn{2}{|c|}{$\begin{array}{c}0-15 \\
15-30 \\
30-45\end{array}$}} & $25.732 \pm 0.723$ & $28.836 \pm 0.502$ & $33.405 \pm 1.884$ & $35.049 \pm 1.684$ & $28.406 \pm 0.266$ \\
\hline & & & $26.439 \pm 0.767$ & $28.279 \pm 0.762$ & $33.991 \pm 2.076$ & $35.311 \pm 1.733$ & $28.048 \pm 0.344$ \\
\hline & & & $26.056 \pm 0.067$ & $27.457 \pm 0.751$ & $34.223 \pm 2.152$ & $35.354 \pm 1.776$ & $27.657 \pm 0.293$ \\
\hline & \multirow{2}{*}{$\begin{array}{c}\text { Combined } \\
0-45\end{array}$} & an & $26.076 \pm 0.315$ & $28.191 \pm 0.366$ & $33.873 \pm 1.028$ & $35.238 \pm 0.959$ & $28.037 \pm 0.172$ \\
\hline & & & 23.974 to 28.227 & 26.202 to 30.332 & 28.007 to 38.040 & 22.980 to 42.127 & 25.202 to 29.332 \\
\hline \multirow{5}{*}{ Silt (\%) } & & $39.900 \pm 2.939$ & $27.378 \pm 0.498$ & $29.998 \pm 1.302$ & $31.359 \pm 1.705$ & $27.671 \pm 0.280$ \\
\hline & \multicolumn{2}{|c|}{$15-30$} & $38.356 \pm 2.557$ & $26.900 \pm 1.268$ & $28.412 \pm 1.209$ & $31.102 \pm 1.643$ & $27.569 \pm 0.593$ \\
\hline & \multicolumn{2}{|c|}{$30-45$} & $42.748 \pm 1.177$ & $28.430 \pm 0.641$ & $29.799 \pm 1.854$ & $30.864 \pm 1.719$ & $28.258 \pm 0.332$ \\
\hline & & ean & $40.335 \pm 1.265$ & $27.569 \pm 0.457$ & $29.403 \pm 0.765$ & $31.108 \pm 0.936$ & $27.833 \pm 0.234$ \\
\hline & & & 32.018 to 46.024 & 23.947 to 29.908 & 25.994 to 36.008 & 24.026 to 49.018 & 17 to 28 \\
\hline \multirow{5}{*}{$\begin{array}{r}\text { Clay } \\
(\%)\end{array}$} & \multicolumn{2}{|c|}{$0-15$} & $34.368 \pm 2.753$ & $43.786 \pm 0.034$ & $35.778 \pm 0.587$ & $33.591 \pm 1.295$ & $43.923 \pm 0.107$ \\
\hline & \multicolumn{2}{|c|}{$15-30$} & $35.204 \pm 2.524$ & $44.821 \pm 0.595$ & $36.196 \pm 0.493$ & $33.587 \pm 1.287$ & $44.383 \pm 0.297$ \\
\hline & \multicolumn{2}{|c|}{$30-45$} & $31.196 \pm 1.189$ & $43.874 \pm 0.818$ & $35.979 \pm 0.543$ & $33.782 \pm 1.347$ & $44.086 \pm 0.364$ \\
\hline & \multirow{2}{*}{$\begin{array}{c}\text { Combined } \\
0-45\end{array}$} & & $33.589 \pm 1.216$ & $44.160 \pm 0.311$ & $35.984 \pm 0.276$ & $33.654 \pm 0.725$ & $44.131 \pm 0.153$ \\
\hline & & & 28.130 to 42.006 & 41.860 to 45.878 & 33.988 to 38.002 & 28.002 to 46.955 & 40.860 to 44.878 \\
\hline \multirow{5}{*}{$\begin{array}{c}\text { Large } \\
\text { Macroaggregate } \\
\text { (\%) }\end{array}$} & \multirow{3}{*}{\multicolumn{2}{|c|}{$\begin{array}{c}0-15 \\
15-30 \\
30-45\end{array}$}} & $7.209 \pm 1.157$ & $34.363 \pm 3.056$ & $58.646 \pm 10.485$ & $16.018 \pm 4.512$ & $21.256 \pm 4.896$ \\
\hline & & & $17.584 \pm 7.184$ & $42.381 \pm 8.588$ & $3 \pm 13$ & $31.780 \pm 7.736$ & $26.911 \pm 6.345$ \\
\hline & & & $11.757 \pm 4.364$ & $38.712 \pm 9.521$ & $54.002 \pm 18.433$ & $61.267 \pm 8.789$ & $25.857 \pm 6.492$ \\
\hline & \multirow{2}{*}{$\begin{array}{c}\text { Combined } \\
0-45\end{array}$} & ean & $12.183 \pm 2.854$ & $38.486 \pm 4.095$ & $51.884 \pm 7.946$ & $36.355 \pm 4.923$ & $24.675 \pm 3.338$ \\
\hline & & & 2.678 to 41.156 & 16.636 to 63.158 & 10.658 to 97.285 & 0.926 to 95.223 & 2.582 to 63.158 \\
\hline \multirow{4}{*}{$\begin{array}{c}\text { Macroaggregate } \\
(\%)\end{array}$} & \multirow{3}{*}{\multicolumn{2}{|c|}{$\begin{array}{c}0-15 \\
15-30 \\
30-45\end{array}$}} & $32.841 \pm 5.076$ & $25.075 \pm 3.019$ & $12.228 \pm 3.091$ & $31.338 \pm 2.306$ & $28.325 \pm 3.519$ \\
\hline & & & $18.779 \pm 2.784$ & $23.320 \pm 4.197$ & $20.204 \pm 5.178$ & $26.167 \pm 3.344$ & $25.048 \pm 3.011$ \\
\hline & & & $25.174 \pm 5.475$ & $22.428 \pm 4.802$ & $18.569 \pm 5.674$ & $13.487 \pm 3.035$ & $25.162 \pm 4.072$ \\
\hline & $\begin{array}{c}\text { Combined } \\
0-45\end{array}$ & $\begin{array}{c}\text { Mean } \\
\text { Range }\end{array}$ & $\begin{array}{c}25.598 \pm 2.776 \\
7.263 \text { to } 63.811\end{array}$ & $\begin{array}{c}23.608 \pm 2.259 \\
7.878 \text { to } 51.601\end{array}$ & $\begin{array}{c}17.000 \pm 2.739 \\
0.312 \text { to } 51.540\end{array}$ & $\begin{array}{c}23.664 \pm 1.841 \\
0.339 \text { to } 56.129\end{array}$ & $\begin{array}{c}26.178 \pm 2.027 \\
7.268 \text { to } 70.460\end{array}$ \\
\hline \multirow{5}{*}{$\begin{array}{c}\text { Microaggregate } \\
(\%)\end{array}$} & & $6.777 \pm 1.106$ & $3.895 \pm 0.501$ & $4.225 \pm 1.609$ & $5.326 \pm 0.469$ & $5.544 \pm 0.725$ \\
\hline & \multicolumn{2}{|c|}{$15-30$} & $8.821 \pm 1.318$ & $2.827 \pm 0.554$ & $4.147 \pm 1.000$ & $3.971 \pm 0.330$ & $5.880 \pm 1.081$ \\
\hline & \multicolumn{2}{|c|}{$30-45$} & $8.300 \pm 1.294$ & $4.321 \pm 0.676$ & $3.003 \pm 1.111$ & $2.947 \pm 0.287$ & $6.012 \pm 0.711$ \\
\hline & ined & Mean & $7.966 \pm 0.714$ & $3.681 \pm 0.341$ & $3.792 \pm 0.723$ & $4.082 \pm 0.224$ & $5.812 \pm 0.490$ \\
\hline & & & 0.307 to 22.618 & 0.279 to 9.833 & 0.153 to 31.717 & 0.041 to 17.447 & 0.279 to 23.271 \\
\hline
\end{tabular}

Tabla 2. Descripción de la textura del suelo y agregados en diferentes lugares y profundidades. Se proporcionan la media y rango, por separado, para profundidades de $0-45 \mathrm{~cm}$; tamaño de agregados del suelo en $\mathrm{mm}$.

Table 2. Description of soil texture and aggregate at different sites and soil depths. Separate mean and range is given for $0-45 \mathrm{~cm}$ depth; size of soil aggregate in $\mathrm{mm}$.

\section{Vegetation analysis at different sites}

For herbs maximum species richness (Table 4) was found at site four (22) followed by site three while minimum at site two (five). Total density was found to be maximum (17.018 plants $\left.\mathrm{m}^{-2}\right)$ at site four followed by site one (9.588 plants $\mathrm{m}^{-2}$ ). Minimum density was found at site two and five (2.771 and 2.721 plants $\mathrm{m}^{-2}$ ). For shrubs/trees maximum species richness was found at site three and four ( 8 species each) followed by site two and five. Density was maximum at site one and four ((8.875 and 8.617 plants $\left.10 \mathrm{~m}^{-2}\right)$ while minimum at site two and five (4.688 and 4.719 plants $10 \mathrm{~m}^{-2}$ ).

\section{Inter-relation of Soil and Vegetation}

Species richness and density for herbs and shrubs/tree was high at site four, with high amount of OC (0.684\%), OM (1.179\%), N (0.059 \%), P (42.338 $\left.\mathrm{kg} \mathrm{ha}^{-1}\right), \mathrm{Ca}\left(170.732 \mathrm{mg} \mathrm{kg}^{-1}\right)$ and $\mathrm{Fe}\left(32.016 \mathrm{mg} \mathrm{kg}^{-1}\right)$ however low amount of clay $(33.654 \%)$, EC $\left(9.441 \mathrm{dSm}^{-1}\right)$ and $\mathrm{Na}$ (68.699 $\left.\mathrm{mg} \mathrm{kg}^{-1}\right)$ was found.

Density of herbs and shrubs/trees was low at site two and five with high amount of clay (44.160 and $44.131 \%), \mathrm{Na}$ (166.149 and 141.128 $\left.\mathrm{mg} \mathrm{kg}^{-1}\right)$ and EC (14.031 and $\left.10.466 \mathrm{dSm}^{-1}\right)$ while low amount of $\mathrm{Ca}\left(51.989\right.$ and $\left.91.120 \mathrm{mg} \mathrm{kg}^{-1}\right)$ and $\mathrm{Fe}\left(19.577\right.$ and $\left.25.740 \mathrm{mg} \mathrm{kg}^{-1}\right)$. Density of herbs and shrubs/trees with clay $(-0.857$ and $-0.903)$ and sodium $(-0.815$ and -0.822$)$ were negative correlated (Fig. 3) while positive correlated with calcium $(0.861$ and 0.952$)$. 


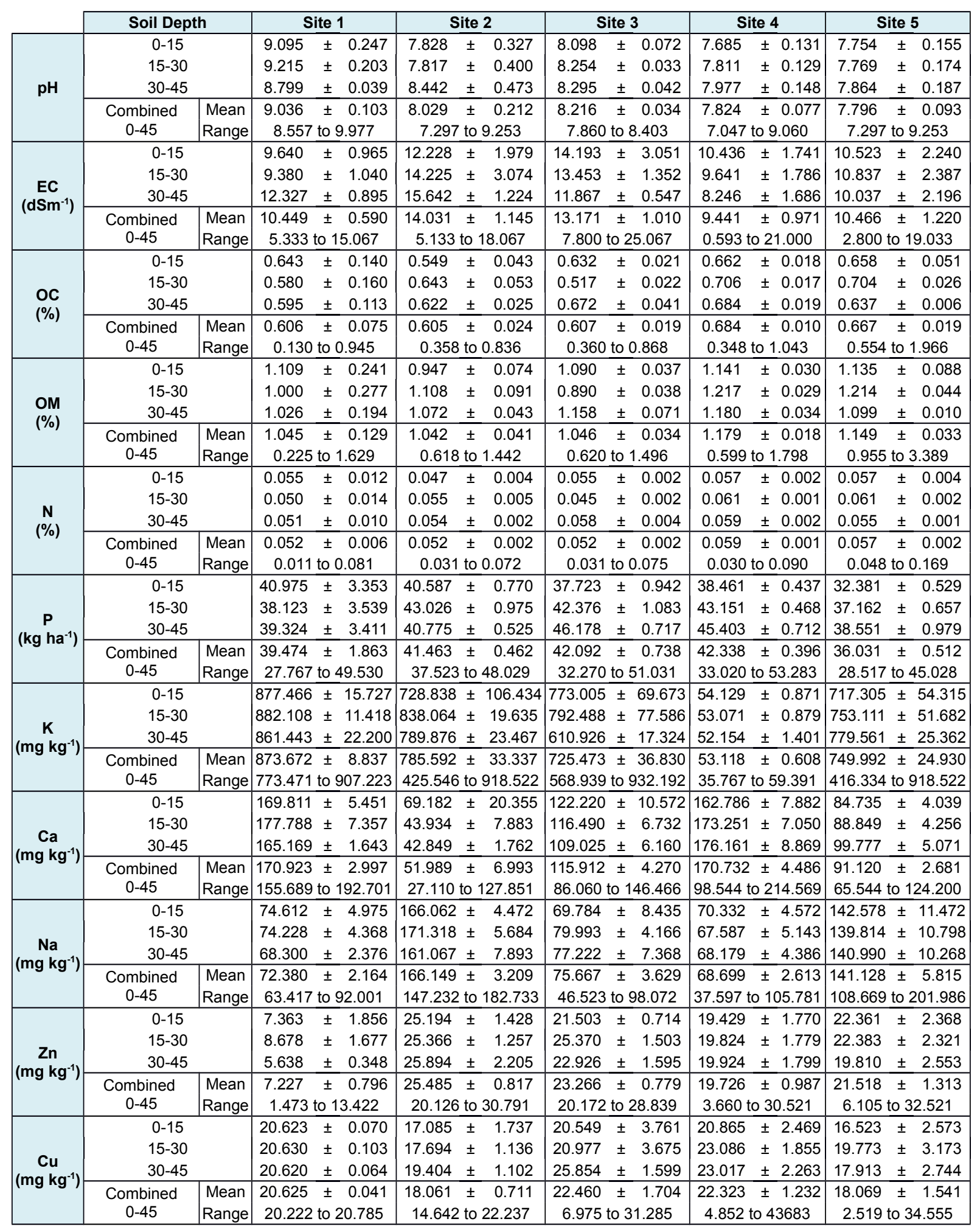

Tabla 3 (sigue). Descripción de propiedades químicas del suelo en diferentes lugares y profundidades. $\mathrm{EC}=$ Conductividad eléctrica, $\mathrm{OC}=$ Carbono orgánico, $\mathrm{OM}=$ Materia orgánica, $\mathrm{N}=$ Nitrógeno total, $\mathrm{P}=$ Fósforo disponible, $\mathrm{K}=$ Potasio, $\mathrm{Ca}=\mathrm{Calcium}$ total, $\mathrm{Na}=\mathrm{Sodio}, \mathrm{Zn}=\mathrm{Zinc}$, $\mathrm{Cu}=$ Cobre, $\mathrm{Fe}=$ Hierro, $\mathrm{Pb}=$ plomo, $\mathrm{Mn}=$ Manganeso; se proporcionan la media y rango, por separado, para profundidades de $0-45 \mathrm{~cm}$.

Table 3 (continues). Description of chemical properties of soil at different sites and soil depths. $\mathrm{EC}=$ Electrical conductivity, $\mathrm{OC}=\mathrm{Organic}$ carbon, $\mathrm{OM}=$ Organic matter, $\mathrm{N}=$ Total nitrogen, $\mathrm{P}=$ Available phosphorous, $\mathrm{K}=$ Potassium, $\mathrm{Ca}=$ Total calcium, $\mathrm{Na}=\mathrm{Sodium}, \mathrm{Zn}=\mathrm{Zinc}, \mathrm{Cu}=$ Copper, $\mathrm{Fe}=\mathrm{Iron}, \mathrm{Pb}=\mathrm{Lead}, \mathrm{Mn}=$ Manganese; separate mean and Range is given for $0-45 \mathrm{~cm}$ depth. 


\begin{tabular}{|c|c|c|c|c|c|c|c|}
\hline & \multicolumn{2}{|c|}{ Soil Depth } & Site 1 & Site 2 & Site 3 & Site 4 & Site 5 \\
\hline \multirow{4}{*}{$\begin{array}{c}\mathrm{Fe} \\
\left(\mathrm{mg} \mathrm{kg}^{-1}\right)\end{array}$} & \multirow{3}{*}{\multicolumn{2}{|c|}{$\begin{array}{c}0-15 \\
15-30 \\
30-45\end{array}$}} & $25.868 \pm 0.504$ & $17.201 \pm 0.766$ & $46.517 \pm 5.933$ & $33.344 \pm 2.923$ & $26.845 \pm 3.577$ \\
\hline & & & $27.184 \pm 1.760$ & $20.735 \pm 4.645$ & $46.255 \pm 5.450$ & $31.367 \pm 2.802$ & $25.995 \pm 3.217$ \\
\hline & & & $24.983 \pm 0.296$ & $20.796 \pm$ & $51.386 \pm 5.506$ & $31.336 \pm 2.610$ & $24.382 \pm 2.948$ \\
\hline & Combined 0-45 & \begin{tabular}{c|} 
Mean \\
Range \\
\end{tabular} & $\begin{array}{c}26.012 \pm 0.584 \\
24.428 \text { to } 34.200\end{array}$ & $\begin{array}{c}19.577 \pm 1.692 \\
15.083 \text { to } 34.496 \\
\end{array}$ & $\begin{array}{c}48.053 \pm 2.890 \\
32.212 \text { to } 60.896\end{array}$ & $\begin{array}{l}32.016 \pm 1.545 \\
11.501 \text { to } 53.327\end{array}$ & $\begin{array}{c}25.740 \pm 1.754 \\
10.031 \text { to } 40.819\end{array}$ \\
\hline \multirow{4}{*}{$\begin{array}{c}\mathrm{Pb} \\
\left(\mathrm{mg} \mathrm{kg}^{-1}\right)\end{array}$} & \multirow{3}{*}{\multicolumn{2}{|c|}{$\begin{array}{c}0-15 \\
15-30 \\
30-45\end{array}$}} & $40.476 \pm 6.987$ & $43.486 \pm 6.597$ & $51.053 \pm 13.867$ & $46.008 \pm 4.266$ & $51.691 \pm 8.524$ \\
\hline & & & $49.732 \pm 5.648$ & $43.396 \pm$ & $37.323 \pm 6.685$ & $43.507 \pm 5.023$ & $44.202 \pm 4.837$ \\
\hline & & & $40.280 \pm 5.390$ & $47.640 \pm$ & $40.421 \pm 8.638$ & $47.849 \pm 5.051$ & $50.635 \pm 7.048$ \\
\hline & $\begin{array}{l}\text { Combined } \\
0-45\end{array}$ & \begin{tabular}{|l|} 
Mean \\
Range
\end{tabular} & 27.198 to 60.218 & 24.287 to 59.659 & $\begin{array}{c}42.932 \pm 5.326 \\
24.524 \text { to } 97.829\end{array}$ & $\begin{array}{l}45.788 \pm 2.666 \\
16.336 \text { to } 94.303\end{array}$ & $\begin{array}{c}48.843 \pm 3.783 \\
13.425 \text { to } 99.869\end{array}$ \\
\hline \multirow{4}{*}{$\begin{array}{c}M n \\
\left(\mathrm{mg} \mathrm{kg}^{-1}\right)\end{array}$} & \multicolumn{2}{|l|}{$0-15$} & $36.012 \pm 3.056$ & $31.093 \pm 6.066$ & $38.164 \pm 1.929$ & $24.637 \pm 2.342$ & $32.430 \pm 3.035$ \\
\hline & \multicolumn{2}{|l|}{$15-30$} & $30.667 \pm 3.158$ & $30.124 \pm$ & $40.139 \pm 1.195$ & $25.970 \pm 2.011$ & $31.548 \pm 2.691$ \\
\hline & \multicolumn{2}{|l|}{$30-45$} & $31.363 \pm 1.792$ & $37.253 \pm$ & $37.392 \pm 2.873$ & $23.791 \pm 2.213$ & $30.786 \pm 2.740$ \\
\hline & $\begin{array}{l}\text { ombined } \\
0-45\end{array}$ & $\begin{array}{l}\text { Mean } \\
\text { Range }\end{array}$ & $\begin{array}{c}32.681 \pm 1.499 \\
24.330 \text { to } 47.057\end{array}$ & $\begin{array}{c}32.823 \pm 2.553 \\
13.353 \text { to } 41.704\end{array}$ & $\begin{array}{l}38.565 \pm 1.100 \\
31.154 \text { to } 46.250\end{array}$ & $\begin{array}{c}24.799 \pm 1.221 \\
7.825 \text { to } 40.343\end{array}$ & $\begin{array}{c}31.588 \pm 1.518 \\
13.353 \text { to } 41.704\end{array}$ \\
\hline
\end{tabular}

Tabla 3. Descripción de propiedades químicas del suelo en diferentes lugares y profundidades. EC $=\mathrm{Conductividad} \mathrm{eléctrica,} \mathrm{OC}=\mathrm{Carbono}$ orgánico, $\mathrm{OM}=$ Materia orgánica, $\mathrm{N}=$ Nitrógeno total, $\mathrm{P}=$ Fósforo disponible, $\mathrm{K}=$ Potasio, $\mathrm{Ca}=\mathrm{Calcium}$ total, $\mathrm{Na}=\mathrm{Sodio}, \mathrm{Zn}=\mathrm{Zinc}, \mathrm{Cu}=$ Cobre, $\mathrm{Fe}=$ Hierro, $\mathrm{Pb}=$ plomo, $\mathrm{Mn}=$ Manganeso; se proporcionan la media y rango, por separado, para profundidades de $0-45 \mathrm{~cm}$.

Table 3 (continued). Description of chemical properties of soil at different sites and soil depths. EC $=$ Electrical conductivity, OC $=$ Organic carbon, $\mathrm{OM}=$ Organic matter, $\mathrm{N}=$ Total nitrogen, $\mathrm{P}=$ Available phosphorous, $\mathrm{K}=$ Potassium, $\mathrm{Ca}=\mathrm{T}$ tal calcium, $\mathrm{Na}=\mathrm{Sodium}, \mathrm{Zn}=\mathrm{Zinc}, \mathrm{Cu}=$ Copper, $\mathrm{Fe}=$ Iron, $\mathrm{Pb}=$ Lead, $\mathrm{Mn}=$ Manganese; separate mean and Range is given for $0-45 \mathrm{~cm}$ depth.

\section{Discussion}

Plant species differ in their sensitivity or tolerance to salts (Brady and Weil 1996). According to Roy et al. (1973) in the soil of Thar Desert of Rajasthan (India) clay content varies from 2 to $6 \%$ in the surface soil and 4 to $8 \%$ in the sub soil. Organic carbon content is very low, ranging from 0.08 to $0.20 \%$ in the surface layer. Moisture retention capacity is very low and the soils are highly pervious. The surface, however, has a tendency to form a crust resulting in reduced infiltration. In this region calcium carbonate increases with the depth. In present study average clay content $(38.303 \%)$ was higher than silt $(31.249 \%)$ and sand $(30.283 \%)$ content. Organic carbon content ranges from 0.130 to $1.966 \%$ and mean value were $0.633 \%$, this shows that saline (Little Rann of Kutch) and Thar Desert are not similar in soil texture and chemical constitutes.

At earlier study it was found that temperature and rainfall affects the salinity and vegetation of the soil (Pilania \& Panchal 2013) of an area. Monsoon has a positive effect on the vegetation and soil properties. While natural hardy vegetation near the surface soil dries up in the dry season, deeper parts of its roots are sustained by moisture supply from the deep soil and with the first rains of the monsoon the desert springs up into life.
Ramakrishna et al. (1966) have discussed in detail about the annual moisture regime variability and its impact on agriculture in Rajasthan desert.

Carbon and nitrogen decreases with the depth which shows conformations with the findings of Charley and West (2010) at semi desert of Utah; because more litter is added from the canopy and surface roots to the surface soil.

In this study plant density was found to be low with respect to high bulk density. The increase of soil bulk density is considered as an important early indicator of ecosystem degradation (Rubio and Bochet 1998) because it leads to further alteration of soil properties such as soil water infiltration and retention (Salihi and Norton 1987). In this area BD and PD were found to be high due to high content of clay and sodium. Highly saline and sodium induced soil reduces amount of water to pass through the root zone regardless of the amount of water actually in the root zone. The high $\mathrm{Na}$ concentration of a sodic soil not only injures plants directly but also degrades the soil. Due to salinity fine particles bind into aggregates. Due to high concentration of sodium in soil, clay platelet, soil dispersion and aggregate swelling takes place. This soil dispersion causes clay particles to close soil pores, which results to reduce soil permeability. Soil dispersion hardens soil and blocks water infiltration, making it diffi- 

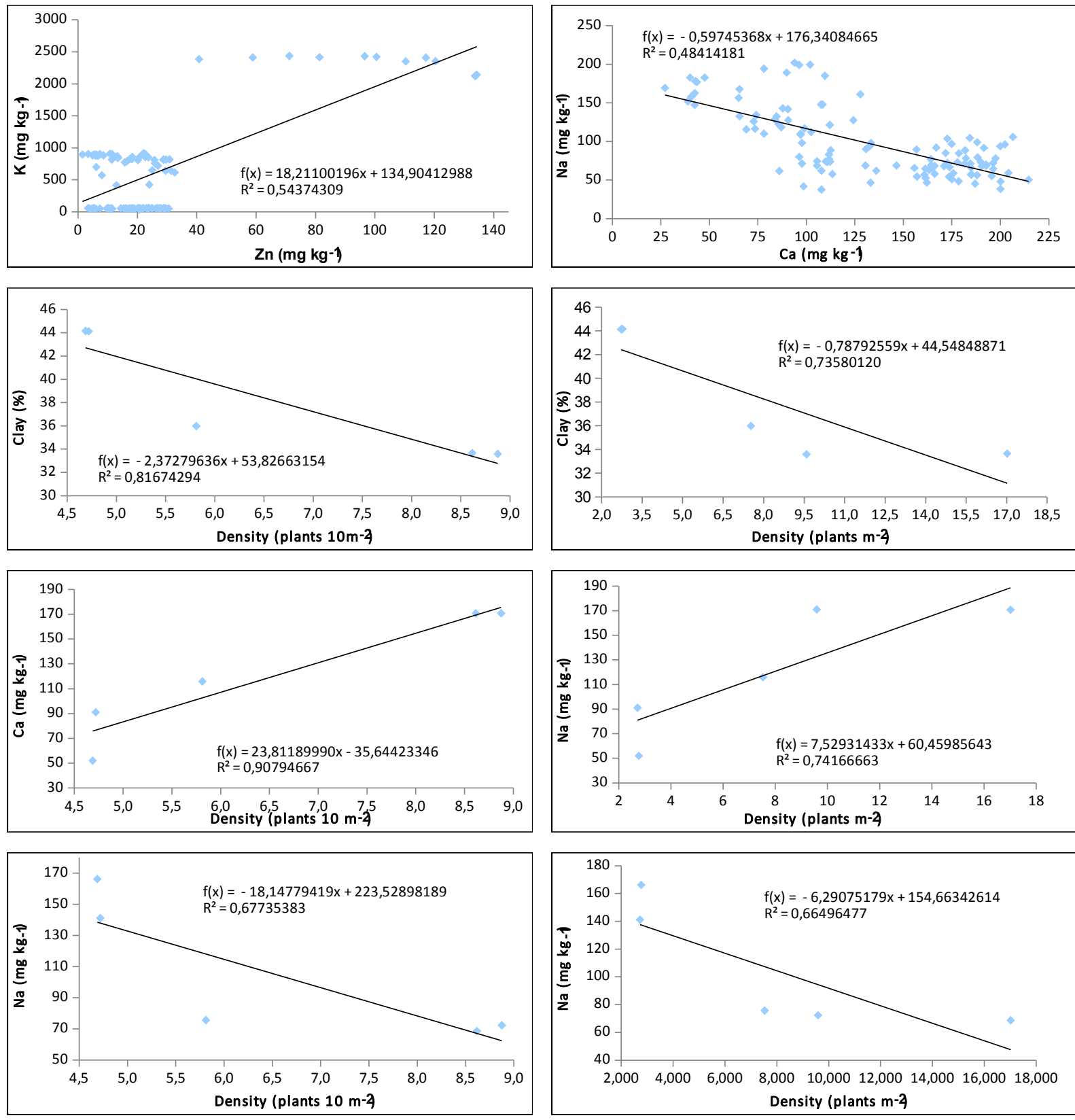

Figura 3. Correlación entre diferentes parámetros del suelo y vegetación en el Pequeño Rann de Kutch i.e. desierto salino.

Figure 3. Correlation between different parameters of soil and vegetation at Little Rann of Kutch i.e. saline desert.

cult for plants to establish and grow (Pilania et al. 2014c)

$\mathrm{EC}$ is the outcomes of the ions and it rises according to the content of soluble salts. EC is directly related to the soluble salts concentration of the soil like $\mathrm{Na}$ and $\mathrm{Mg}$ (Maiti 2003). High value of EC and high percentage of clay affects vegetation negatively and are harmful for vegetation (Pilania \& Panchal 2014) and the same type of negative effect were found during this study. Panchal \& Pandey (2002) mentioned that soil salinity increases with soil degradation or deserti- fication. Spatial variability of soil physical and chemical properties at a large scale is mainly due to geological, geomorophological and pedological soil forming factors that could be altered and induced by other factors such as land use managements. Parejiya et al. (2015) found approx 20 species for each studied site at Bandiyabedi forest grassland of Surendranagar district in Gujarat (India); Pilania et al. (2014a) documented 65 species of 57 genera belonging to 31 families at Tropical dry deciduous forest of Dahod district of Gujarat and Pilania et al. (2014b) documented 80 


\begin{tabular}{|c|c|c|c|c|c|}
\hline & Site 1 & Site 2 & Site 3 & Site 4 & Site 5 \\
\hline \multicolumn{6}{|c|}{ Herbs } \\
\hline $\begin{array}{c}\text { Total Density } \\
\left(\text { plants } \mathrm{m}^{-2} \text { ) }\right. \\
\text { Total Species }\end{array}$ & $\begin{array}{c}9.588 \pm 1.23 \\
6\end{array}$ & $\begin{array}{c}2.771 \pm 1.37 \\
5\end{array}$ & $\begin{array}{c}7.531 \pm 0.65 \\
9\end{array}$ & $\begin{array}{c}17.018 \pm 0.86 \\
22\end{array}$ & $\begin{array}{c}2.721 \pm 0.78 \\
8\end{array}$ \\
\hline \multicolumn{6}{|c|}{ Shrubs / Trees } \\
\hline $\begin{array}{l}\text { Total Density } \\
\text { (plants } 10 \mathrm{~m}^{-2} \text { ) } \\
\text { Total Species }\end{array}$ & $\begin{array}{c}8.875 \pm 0.65 \\
3\end{array}$ & $\begin{array}{r}4.688 \pm 0.4 \\
11\end{array}$ & $\begin{array}{c}5.813 \pm 0.48 \\
2\end{array}$ & $\begin{array}{c}8.617 \pm 0.88 \\
5\end{array}$ & $\begin{array}{c}4.719 \pm 0.34 \\
2\end{array}$ \\
\hline
\end{tabular}

Tabla 4. Análisis de la vegetación en los distintos lugares del Pequeño Rann de Kutch i.e. desierto salino. Table 4. Vegetation analysis at different sites of Little Rann of Kutch i.e. Saline Desert.

species belonging to 37 families at home gardens of South Gujarat; which shows that this saline desert have low species richness so major steps are required to increase the vegetation.

At site one and four high concentration of $\mathrm{Ca}$ (170.923 and $170.732 \mathrm{mg} \mathrm{kg}^{-1}$ ) and low $\mathrm{Na}$ (72.380 and $68.699 \mathrm{mg} \mathrm{kg}^{-1}$ ) was found with maximum plant density, which suggests that $\mathrm{Na}$ have negative effects on salinity. The application of gypsum has long been considered a common exercise in reclamation of saline sodic and sodic soils (Marschner 1995). The addition of calcium to the soil (as lime or gypsum) displaces $\mathrm{Na}^{+}$from clay particles. This prevents the clay from swelling and dispersing (Sumner 1993) and also makes it possible for $\mathrm{Na}^{+}$to be leached deeper into the soil. Thus, exogenously supplied calcium not only improves soil structure, but also alters soil properties in various ways (Shabala et al. 2003) that benefit the plant growth. Moreover, an improved $\mathrm{Ca} / \mathrm{Na}$ ratio in the soil solution enhances the capacity of roots to restrict $\mathrm{Na}^{+}$influx (Marschner 1995). Importance of interaction between $\mathrm{Na}$ and $\mathrm{Ca}$ was recognized after LaHaye and Epstein (1969) reported that exogenously supplied calcium may significantly alleviate detrimental effects of $\mathrm{Na}^{+}$on the physiological performance of hydroponically grown plants.

\section{Conclusion}

Soil with low concentration of OC, OM, N, P, Fe, $\mathrm{Ca}$ and high concentration of clay, $\mathrm{Na}$ and $\mathrm{EC}$ cause low species richness and density. Emergent of native and dominant species like Cressa cretica Linn., Capparis deciduas (Forsk.) Edgew., Acacia nilotica (Linn.) Del, etc. at fringe vicinity with furnishing necessary nutrients can facilitate to en- hance green belt, improve soil structure and help to control the extension of desert condition.

\section{References}

Bouyoucos GJ. 1951. A recalibration of the Hydrometer Method for Making Mechanical Analysis of soils. Agronomy Journal, 43: 434-438.

Brady NC \& Weil RR. 1996. The Nature and Properties of Soils. Englewood Cliffs, NJ: Prentice-Hall.

Charley JL \& West NE. 2010. Plant-induced soil chemical patterns in some shrub-dominated semi-desert ecosystems of Utah. Journal of Ecology 63(3): 945963.

Curtis JT \& Mclntosh RP. 1950. The interrelations of certain analytic and synthetic phytosociological characters. Ecology 31: 434-455.

Donahue RL, Miller RW \& Shickluna JC. 1983. Soils: An Introduction to Soils and Plant Growth. Englewood Cliffs, NJ: Prentice-Hall.

Garg BK \& Gupta IC. 1997. Saline Wastelands Environment and Plant Growth. Jodhpur, India: Scientific Publishers.

Jackson ML 1973. Soil chemical Analysis. New Delhi: Prentice.

Jenny H. 1941. Factors of Soil Formation. NewYork: Mc.Graw Hill Publishers.

La Haye PA. \& Epstein E. 1969. Salt toleration by plants: enhancement with calcium. Science 166: 395-396.

Lindsay WL \& Norvell AW. 1978. Development of DTPA soil test for zinc, iron, manganese and copper. Soil Science society America Journal 12: 421-428.

Maiti SK. 2003. Handbook of methods in Environmental Studies. Vol. 2: Air, Noise, Soil and Overburden Analysis. Jaipur: ABD Publishers.

Margalef R. 1958. Perspective in ecological theory. Chicago: University of Chicago Press.

Marschner H. 1995. Mineral Nutrition of Higher Plants. London: Academic Press.

Mer RK, Prajith PK, Pandya DH \& Pandey AN. 2000. Effect of salts on germination of seeds and growth of young plants of Hordeum vulgare, Triticum aestivum, Cicer arietinum and Brassica juncea. Journal of Agronomy and Crop Science 185: 209-217. 
Misra R. 1968. Ecology Workbook. New Delhi: Oxford and IBH Publishing Co.

Morgan RPC. 1996. Soil erosion and conservation. Silsoe College, Cranfield University.

Nearing MA, Jetten V, Baffaut C, Cerdan O, Couturier A, Hernandez M, Bissonnais Y, Nichols MH, Nunes JP, Renschler CS, Souchère V \& Oost K. 2005. Modeling response of soil erosion and runoff to changes in precipitation and cover. Catena 61(2-3): 131-154.

Olsen SR, Cole CV, Watanabe FS \& Dean LA. 1954. Estimation of available phosphorus in soils by extraction with sodium bicarbonate. Washington DC: US Department of Agriculture.

Panchal NS \& Pandey AN. 2002. Study on Soil Properties and their influence on vegetation in Western Region of Gujarat State in India. 12th ISCO Conference, Beijing 2002: 610-615.

Parejiya NB, Detroja SS \& Panchal NS. 2015. Analysis of herbaceous vegetation of Bandiyabedi forest grassland of Surendranagar district in Gujarat (India). International Journal of Science \& Medical Research 2: 28-46.

Pilania PK \& Panchal NS. 2013. Vegetation analysis at Little Rann of Kachchh in Mallya Tehsil of Gujarat state in India. Journal of Basic and Applied Biology 7: 6-10.

Pilania P.K. \& Panchal N.S. 2014. Soil and plant relation at Little Rann of Kutch of Gujarat in western India, International Journal of Advanced Research, 2:7, 110.

Pilania PK, Gujar RV \& Panchal NS. 2014a. Species diversity and phytosociological analysis of important plants of Tropical Dry deciduous forest of Dahod district of Gujarat, India. International Journal of Science \& Medical Research, 1:37-46.

Pilania P.K., Gujar R.V., Bhatt J.D., Joshi P.M., Joshi A.B., Shrivastav S.C. \& Panchal N.S. 2014b. Tree diversity and utilities in homegardens of south Gujarat, Western india: an overview. International Journal of Science \& Medical Research 1: 53-59.

Pilania PK, Vaghasiya PM, Panera NM, Mirani MK \& Panchal NS. 2014c. Ecological study at Morbi district near Little Rann of Kachchh in western India, International Journal of Advanced Research 2(4): 513.

Postel S. 1996. Forging a sustainable water strategy. In: State of the world 1996: A Worldwatch Institute re- port on progress toward a sustainable society (Brown RL, ed.) New York: W W Norton \& Co Inc, pp. 40-59.

Ramakrishna YS, Victor US \& Ramana-Rao BV. 1966. In: Climate Variability and agriculture (Abrol YP, Gadgil S \& Pant GB, eds), New Delhi: Narosa, pp. 242-252.

Refahi HG. 2006. Soil erosion by water \& conservation. Tehran: University of Tehran press.

Roy BB, Dhir RP \& Kolarkar AS. 1973. Soils of Rajasthan and their characteristics. Proceedings of the Indian national Science Academy 44(4): 161-167.

Rubio JL \& Bochet E. 1998. Desertification indicators as diagnosis criteria for desertification risk assessment in Europe. Journal of Arid Environment 39: 113-120.

Salihi DO \& Norton BE. 1987. Survival of perennial grass seedling under intensive grazing in semiarid rangelands. Journal of Applied Ecology 24:145-151.

Shabala S, Shabala L \& Volkenburgh EV. 2003. Effect of calcium on root development and root ion fluxes in salinised barley seedlings. Functional Plant Biology 30: 507-514.

Sumner M.E. 1993. Sodic soils: new perspectives. Australian Journal of Plant Physiology 31:c683-750.

Thornes J. 1996. Desertification in the Mediterranean. In: Mediterranean desertification and land use (Brandt J \& Thornes J, eds.). Chichester, England: Wiley, pp. 1-12.

Trimble S.W. 1990. Geomorphic effects of vegetation cover and management: Some time and space considerations in prediction of erosion and sediment yield. In: Vegetation and erosion, processes and environments (Thornes J, ed.). Wiley, London, pp. 5565.

Richards, LA (ed.) 1968. Diagnosis and improvement of saline and alkaline soils. Agriculture Handbook 60. Washington DC: US Department of Agriculture.

Vaghasiya PM, Pilania PK, Panera NM, Mirani MK \& Panchal NS. 2015. Analysis of vegetation at Saline desert (Great Rann of Kutch) of Gujarat state in western India. International Journal of Science \& Medical Research 2: 67-83.

Yoder RE. 1936. A direct method of aggregate analysis of soils and a study of the physical nature of erosion losses. Journal of American Society of Agronomy 28: 337-351. 